\title{
COVID-19 Personal Protective Equipment in the Home: Navigating the Complexity of Donning and Doffing
}

\author{
Brian Kerley, MD, CCFP(PC), \\ FCFP ${ }^{1,2}$ \\ Lana Tan, MD, CCFP $(P C)^{2,3}$ \\ Denise Marshall, $M D, C C F P(P C)$, \\ FCFP $^{2,4}$ \\ Cindy O'Neill, MLT, ART, CIC \\ Anne Bialachowski, RN, BN, MS, \\ $\mathrm{CIC}^{6}$ \\ José Pereira, $M B C b B, C C F P(P C)$, \\ $M S c, F C F P^{2,7}$ \\ 'Niagara North Family Health Team, St \\ Catharines, Ontario, Canada \\ ${ }^{2}$ Division of Palliative Care, Department \\ of Family Medicine, McMaster University, \\ Hamilton, Ontario, Canada \\ ${ }^{3}$ Kitchener Waterloo Community Palliative \\ Care Team, Kitchener, Ontario, Canada \\ ${ }^{4}$ Niagara West Palliative Care Team, \\ Grimsby, Ontario, Canada
}

${ }^{5}$ Infection Prevention and Control Program Hamilton Health Sciences, Ontario,

Canada

${ }^{6}$ Infection Prevention and Control Program St Joseph's Healthcare Hamilton, Hamilton, Ontario, Canada

${ }^{7}$ Pallium Canada, Ottawa, Ontario, Canada

Conflicts of interest: authors report none.

\section{CORRESPONDING AUTHOR}

José Pereira, MBChB, CCFP (PC), MSc, FCFP

David Braley Health Sciences Centre 100 Main Street West, 2nd Floor Hamilton, Ontario, Canada L8P $1 \mathrm{H} 6$ jpereira@mcmaster.ca

\begin{abstract}
PURPOSE The safety of care professionals and patients is paramount while caring for people with infectious diseases, including those with confirmed or suspected severe acute respiratory syndrome coronavirus 2 (SARS-CoV-2) infection and coronavirus disease 2019 (COVID-19). Existing policies and protocols for donning and doffing personal protective equipment (PPE) are primarily for institutional settings such as hospitals, not for home visits for patient care. We describe a protocol for donning and doffing PPE in home settings.
\end{abstract}

METHODS We used an iterative, rapid-prototyping approach to develop the protocol. A small workgroup created preliminary drafts, drawing on hospitalbased protocols and modifying them, while undertaking simulations. Wider input was solicited via 2 webinars; 1 regional (Hamilton, Ontario) with palliative clinicians, and 1 national (Canada) with varying professions. We also consulted a group of infectious disease experts. A "how-to" video accompanies the protocol.

RESULTS Twelve versions of the protocol were produced, with major changes occurring within the first 6 versions. A national webinar mid-development provided further validation and minor modifications. Subsequent versions involved minor changes. The protocol has 4 phases: (1) Preparing, (2) Entering the Home, (3) Leaving the Home, and (4) After the Visit and Reprocessing. In addition to PPE-related equipment, the protocol requires additional materials including 2 pails for transporting supplies, plastic bags, hand sanitizer, disinfectant wipes, and printed easy-to-use checklists.

CONCLUSIONS This protocol addresses gaps in COVID-19-related guidelines, specifically the process of donning and doffing PPE during home visits while supplementing jurisdictional PPE guidelines and protocols.

Ann Fam Med 2021;19:405-410. https://doi.org/10.1370/afm.2667.

Appeared as Annals "Online First" article.

\section{INTRODUCTION}

The safety of care professionals and patients is paramount while caring for people with infectious diseases, including those with confirmed or suspected severe acute respiratory syndrome coronavirus 2 (SARS-CoV-2) infection and the accompanying coronavirus disease 2019 (COVID-19). Policies and protocols related to donning and doffing personal protective equipment (PPE) are now widely available, but developed primarily for institutional settings such as hospitals, and thus may not prove useful for clinicians doing home visits for patient care.

The process of donning and doffing PPE is more complex in a home setting compared with other settings of care such as hospitals. In institutional health care settings, necessary PPE equipment (eg, surgical masks or N95 respirators, gowns) is readily available outside patients' rooms, often accompanied by instructional posters, as part of a coordinated effort by the institution's Infection Prevention and Control (IPAC) team. On completion of a patient encounter, there are bins to discard used PPE. Hospital processes handle the soiled reusable and non-reusable equipment, which 
allows the health care professionals to focus on safe donning and doffing and providing care.

In the home care setting, a number of complexities arise. There are no isolation carts or shelves with PPE awaiting outside a patient's house, townhouse, or apartment. There are no colleagues to help oversee the process and ensure safety. Hand sanitizers and disinfectant wipes cannot normally be left outside the door to a house or apartment for a number of reasons, including possible theft. Transporting clean and contaminated medical equipment to and from the home adds another layer of complexity.

In mid-March 2020, in the early days of Canada's COVID-19 pandemic mobilization efforts, a community workgroup was established by the Division of Palliative Care in the Department of Family Medicine at McMaster University in Ontario, Canada. The goal was to mobilize palliative care services and develop resources (including clinical guidelines) $)^{1}$ for the city of Hamilton and surrounding regions in Ontario. During the inaugural meeting, the workgroup identified a need for "howto" guidelines for donning and doffing PPE in the home setting. Searches of the literature and public health websites failed to find such a resource. The objective of this paper is to describe the development of a protocol for donning and doffing PPE in the home and to share the protocol with colleagues who provide home-based care.

\section{METHODS}

Given the urgency of the situation, we used an iterative rapid-prototyping approach to develop a donning and doffing PPE protocol. A small work group (B.K., D.M., L.T., J.P.) developed preliminary drafts, drawing on protocols used by hospitals, long-term care facilities, and public health authorities. The group also ran simulations to refine the protocol. Broad consultation was then solicited via 2 webinars. The webinars enabled sharing the protocol and soliciting further input from end users.

The first webinar on March 25, 2020 was a regional webinar hosted by the work group. Invitations were sent to members of the Division of Palliative Care at McMaster and their team colleagues. The second webinar on April 3, 2020 was national in scope and hosted in partnership with Pallium Canada (www. pallium.ca). Pallium Canada sent out an invitation to its network and community of practice across Canada, which consists of about 900 palliative care clinicians and educators, and over 10,000 health care professionals who had previously participated in Pallium Canada's Learning Essential Approaches to Palliative Care (LEAP). The webinar was also posted on the Pallium website. An electronic survey was sent to all participants of the national webinar, to evaluate the usefulness of the webinar and the protocol. Field notes were chronicled during both webinars, including comments and questions submitted via the chat function. The protocol was further revised based on the field notes and webinar recording. A video recording of the webinar is available at https://www.youtube.com/ watch?v=h34W7yozmWU\&feature=youtu.be.

On May 10, 2020, the provincial body, Public Health Ontario, published new guidelines on the use of PPE and conservation of PPE. We reviewed our protocol to ensure it aligned with the new guidelines, resulting in 1 minor change related to the use of diluted bleach to clean equipment post visit. Lastly, in June 2020 a regional team of infectious disease and Infection Prevention and Control (IPAC) experts from Hamilton Health Sciences and St Joseph's Healthcare provided input, by way of written submissions and conference calls. The final modifications were made by consensus to leverage both the expertise of the IPAC experts and the real-world practical experiences of the palliative care clinicians. This project was not submitted for review by an ethics committee since it represents quality improvement work, constituted an urgent need, and did not involve study participants.

\section{RESULTS}

A total of 12 versions of the protocol were produced, with major changes occurring within the first 6 . The national webinar (at Version 6) resulted in minor modifications, and provided further validation of the protocol. At this point we achieved saturation on major components. Subsequent versions involved minor tweaks, such as the concentration of diluted bleach to clean eyewear, and the addition of new complementary links to emerging public health-related documents such as policies on PPE conservation.

The first webinar had 87 participants, mainly Palliative Division physicians and their nursing colleagues from Hamilton and surrounding regions of southern Ontario. A total of 268 people attended the second national webinar (including 96 registered nurses, 72 physicians, 12 licensed practical nurses, 9 social workers) from 11 provinces and territories. The webinar was recorded and is available online. The brief survey sent to participants of the national webinar solicited 53 responses ( 22 physicians, 22 nurses, 9 unknown, response rate $19.8 \%$ ). Of these responses, 51 (97\%) agreed with the statement that the webinar was relevant to their practice. An emerging theme from the comments posted in the webinars' chat was a greater appreciation of the complexities of donning and doffing of PPE in the home. 
The final (current) version of the protocol (Table 1) is divided into 4 phases: (1) Preparing, (2) Entering the Home, (3) Leaving the Home, and (4) After the Visit and Reprocessing. The protocol details full PPE use (mask, gown, goggles or face-shield, and gloves). It requires the user to apply local or jurisdictional guidelines when to use a surgical mask vs an N95 respirator and how to conserve PPE. In addition to PPE equipment (including spare gloves and masks), the protocol requires two 2- to 3 - gallon pails (1 designated clean and 1 dirty) for transporting supplies, 2 clean plastic garbage bags (one to serve as work surface inside the home and smaller one to carry equipment), a bottle of hand sanitizer, and a container of disinfectant wipes (or a small ziplock bag with several wipes). The protocol assumes the clinician is well versed in hand hygiene.

The entire protocol, including user-friendly checklists (cue sheets), is available online, at https:// fhs.mcmaster.ca/palliativecare/COVID19Resources. html (PPE in the Home section). A supplementary, low-budget explanatory video was produced to help understand and apply the protocol (PPE in the Home section, https://fhs.mcmaster.ca/palliativecare/ COVID19Resources.html).

\section{DISCUSSION}

The complexities of donning and doffing PPE in a home setting are often overlooked or not fully appreciated, despite being considerably greater than in institutional health care settings. This protocol provides a resource for health care professionals providing home visits for general or palliative care, when PPE is needed as per jurisdictional criteria and policies.

Our rapid-prototyping approach worked well because it harnessed quality improvement principles, including an iterative development process while creating a robust product on a short timeline. We were diligent to ensure the first version made widely available (Version 4), was of good quality and did not pose safety risks. The subsequent protocol modifications provided further improvements. The need for speedy responses in pandemic situations has previously been articulated. The Executive Director of the World Health Organization's Emergencies Program, Dr Michael Ryan, stated, "Speed trumps perfection in a pandemic." ${ }^{2}$ We believe our development process allowed for safety and speed, with the webinars providing further input and validation.

While this protocol was developed by palliative care clinicians and infectious disease specialists, its utility is not restricted to palliative care-related home visits. It is applicable for other home visits or home care. It does assume that the patient has reduced functional status and/or is incapacitated, which is often the case in patients receiving end-of-life care (recognizing as we do, that palliative care is not limited to end-of-life care and should be activated much earlier in the illness trajectory).

Additional adjustments to the protocol may be needed to account for local and temporal circumstances. Adaptations may be required for congregate housing environments such as apartments, or modifications for inclement weather conditions such as extreme cold, rain, and snow. In extremely cold weather, a strategy may be needed regarding one's coat if it cannot be taken into the home. Some patients or families may request that care professionals remove their footwear on entering the home so shoe covers could be considered (not included in PPE protocols). Patients and family members may not be comfortable or able to dispose of the garbage bag with any used supplies, so the clinician will need to be accommodating. Further, the clinician may need to modify the exit procedure depending on the configuration of the front door of the home, particularly in phase 3, Step 3 (using a disinfectant wipe to clean the inner door handle before leaving), as some homes have additional screen doors. The question as to whether one could don the PPE in one's workplace before departing for the home visit may appear practical at first glance but warrants further consideration. Firstly, donning PPE so far from the point-of-care may be problematic as the equipment could become contaminated, thereby increasing the risk of introducing the virus into a virus-free home if PPE is being donned in a situation where the patient's virus status is unknown but precautions are being applied. Second, in very cold winter climates such as in Canada and northern states of the United States of America, the wearer would need to don the equipment over very large bulky winter coats, making driving potential risky and the visit in a heated home very uncomfortable.

Clinicians can also make improvisations to the protocol, but may need to exercise even greater caution depending on the change. For example, bins (or boxes) lined with garbage bags could be used instead of pails for transporting clean and dirty supplies. One would need to be cautious, however, when tying the bags to avoid aerosolizing the virus with a puff of air as one gathers the bag. Gloves would be required for closing and opening the bags, with hand hygiene after each step. Further, carrying boxes may be more difficult to carry than pails with handles. The large bag that serves as a working surface at the entrance of the home could be repurposed when exiting the home to bring out the re-usable, wiped-down equipment, instead of using pails that were left outside theentrance 
Table 1. The McMaster Home Visit PPE Procedure for Patients With Respiratory Illnesses - Revised

\section{Phase 1: Preparing}

1. Pre-visit screening: All care professionals should self-monitor before the visit using current provincial and employer screening practices.

2. Conduct a virtual visit or telephone call before going to the home. Do history taking and as much discussion as you can over the telephone or video, so that only a physical exam will take place in the home and whatever discussion is needed as a result of your clinical assessment. Request that a family member or caregiver be in the home to help you exit the home safely afterwards.

3. Ask the family/caregivers to:

- Place a garbage bin lined with a garbage bag just inside the entrance of the home/apartment

- Ensure the patient is wearing a surgical mask when you arrive. The family/caregivers should also put on surgical or cloth masks when you arrive. (Remember to bring extra masks in case they do not have any.)

- If they have hand sanitizer, have the patient do hand hygiene when you arrive

- Keep pets and children in a separate area, away from the front door where you will set up your workstation and ask them to request that other people not visit while you are there

- Try to limit the number of people in the home to the patient and 1 caregiver, if possible, during your visit. Advise them that you will need to maintain physical distancing as much as possible during your visit.

4. Print 2 copies of the Doffing PPE Cue Sheet (https://fhs.mcmaster.ca/palliativecare/ documents/HomeVisitPPEFullProcedurev.12.128July2020.pdf), one for outside the door and one for inside the door.

5. Bring with you:

- A container of alcohol-based hand rub (ABHR)

- A container of disinfectant wipes (or ziplock bag containing 2-3 disinfectant wipes)

- A large plastic garbage bag

- A small plastic bag (for carrying supplies into the home)

- Two empty pails - one labeled "Clean" and one "Dirty" (a lid for the dirty pail would be ideal)

- Extra ear-loop masks for patient and caregivers if they do not have any

- Two copies of the Doffing PPE Cue Sheet

- Your PPEb

- Two to 3 extra sets of gloves in a second ziplock bag

- Diagnostic equipment you need (eg, stethoscope, oximeter)

\section{Phase 2: Entering the Home}

1. Call the house from the driveway and confirm that the family has a mask for the patient and any caregivers in the house. If they do have masks, you do not need to bring spare masks into the home. Ask everyone to don their masks.

2. Place the empty pails outside the door with lid(s) off, with the "Clean" pail closest to the door so that it can be easily reached from the threshold. Put one of the clean cue sheets on the ground next to the pails, secured so it doesn't blow away.

3. Don PPE outside the home.

4. Enter the home carrying the small plastic bag in which you have placed the disinfectant wipes, the spare gloves, the large plastic garbage bag, the $A B H R$, the spare mask (if needed) and your diagnostic equipment.

5. Place the small plastic bag on the floor inside the doorway.

6. Lay the unopened large garbage bag flat on the floor next to the patient's garbage bin just inside of the front of the home. This will serve as a clean work surface. Remove from the small plastic bag the disinfectant wipes, the ABHR, the spare gloves and the second copy of the Doffing PPE Cue Sheet and place these on the clean work surface.

7. Bring the small plastic bag containing your diagnostic equipment and mask for patient (if needed) to the patient's room. Ensure the patient is wearing a mask and does hand hygiene with their own ABHR if they have not already done so. Do not offer your own ABHR for this purpose.

8. Perform physical exam then place the used equipment back in the small plastic bag.

ABHR = alcohol-based hand rub; AGMP = aerosol-generating medical procedures; BIPAP = bilevel positive airway pressure; CPAP = continuous positive airway pressure; DIN = drug identification number; LPM = liters per minute; PPE = personal protective equipment; $N 95$ = respiratory protective device with very close facial fit and very efficient filtration of airborne particles.

${ }^{a}$ ABHR sanitizer must be at least $60 \%$ ethanol.

bPPE in this instance will include an isolation gown, eye protection (goggles, face shield, or a mask with an integrated visor), gloves, and a mask. The previous version of this protocol suggested a medical mask unless AGMPs are being conducted. However, with the growing realization that aerosol transmission of COVID-19 is common, a point-of-care risk assessment must be done, and consideration given to wearing a fit-tested N95 respirator with any patient with a respiratory illness, regardless of vaccination status.

door. The risk of that strategy is the bag could have been in contact with virus from the surface it was laid on (the concern being the potential for fomite spread). In addition, it may be more difficult to hold wiped equipment in one hand while trying to open a closed bag with the other, instead of simply placing the equipment in a pail.

Health care professionals often make multiple home visits each day. In this situation, several sets of PPE are required, 1 for each visit that requires $P P E$ use. If PPE supplies are limited, it may be possible to use the same mask and eye protection for several successive visits, provided the clinician does not touch either item while not wearing gloves, and can safely drive their car with the items on. However, in at least one Canadian province (Ontario), a regulatory authority (Ontario Health) recommends that "once you exit the patient's home you should immediately dispose of the mask in the appropriate receptacle." ${ }^{3}$ Once a mask and eyewear are removed, there is a risk of selfcontamination when they are re-applied, therefore once removed they should be discarded or reprocessed as appropriate. Some improvisations such as the use of plastic containers to store masks when not in use and reapplying them without touching the outside of the mask have been suggested.

We found varying opinions regarding the cleaning of used eye protection (goggles and face shields). 


\section{Phase 3: Leaving the Home}

1. With full PPE still on, bring the small plastic bag with used equipment to the front door. Lay the plastic bag on the floor beside the clean large garbage bag work surface so as not to contaminate the work surface.

2. Perform hand hygiene on your gloves with ABHR (remember, you have now contaminated the ABHR container). Alternatively, gloves may be removed and discarded into the patient's garbage bin, hand hygiene is performed with $A B H R$, then fresh gloves are donned. The spare sets of gloves may also be useful if a glove is torn in the process.

3. With a disinfectant wipe, clean your diagnostic equipment and lay the clean equipment on the large garbage bag. If still wet, use the same disinfectant wipe to clean the container of $A B H R$, the ziplock bag containing the spare gloves, and the inner door handle to the home.

4. After a minute, repeat step 3 with a fresh wipe.

5. Touching only the clean door handle, open the door and drop the clean equipment and ABHR into the "Clean" pail outside the door. Keep the ABHR accessible as you will need it again.

6. Discard the small bag that had contained the equipment and the empty ziplock bag that had contained the disinfectant wipes into the patient's garbage bin.

7. Doff gown and gloves inside the front door and put in the patient's garbage bin. Don't push down.

8. Exit the house touching only the cleaned door handle, leaving behind the Doffing PPE Cue Sheet on the large flat garbage bag.

9. Outside of the door, perform hand hygiene with ABHR.

10.If wearing goggles or face shield with separate mask, remove goggles or face shield by touching only the most posterior parts of the arms, and place in the "Dirty" pail.

11. Remove mask touching only the back of the ear loops and put it in the "Dirty" pail with the eyewear. If you have a used N95 mask, it may be prudent to save your mask, as techniques for reprocessing $\mathrm{N} 95$ masks may be coming. If so, place it in the "Dirty" pail to bring with you for later processing.

12.Put the outdoor copy of the Doffing PPE Cue Sheet in the "Dirty" pail.

13. Hand hygiene with ABHR.

14.Put the lid on the "Dirty" pail. Don't touch the goggles/face shield or mask.

15. Pack your car.

\section{Phase 4: After the Visit and Reprocessing}

1. At your clinical base, don fresh gloves and eye protection. A face shield is ideal. Consider wearing a washable cloth gown also, in case of a splash.

2. Remove already-cleaned equipment from the "Clean" pail, then disinfectant wipe the pail.

3. Prepare a solution of $0.1 \%$ sodium hypochlorite (1 part household bleach to 49 parts water, eg, $50 \mathrm{ml}$ bleach added to $2,450 \mathrm{ml}$ of water).

4. With PPE still on, open the "Dirty" pail, remove any masks and cue sheets and place in garbage, making sure you don't touch the outside of the garbage bin/ box with the masks or gloves as these are considered contaminated. Consider placing N95 masks in a paper bag (such as a large leaf bag) for storage and potential future reprocessing.

5. Put some dish soap and water carefully in the "Dirty" pail so as not to splash.

6. Wash goggles, rinse with water, then place in $0.1 \%$ sodium hypochlorite solution to soak for 10 minutes.

7. Wash out the pail thoroughly with then disinfectant wipe it. Disinfectant wipe the dish soap bottle and your face shield.

8. Remove gloves and dispose in garbage and remove gown if worn).

9. Hand hygiene with ABHR.

Note: We found varying opinions for cleaning of reusable eye protection (goggles and face shields). Some suggest using only disinfectant wipes while others suggest a diluted bleach soak. Health Canada recommends cleaning with soap and water then disinfecting using an approved hard surface disinfectant with a DIN number, or alternatively, a bleach soak. The theoretical advantage of the bleach soak is that it will penetrate the hinges and small parts of the eye protection, and that it conserves disinfectant wipes which may be in short supply. Health Canada states that eyewear may be disinfected (after appropriate cleaning with soap and water) using a $0.1 \%$ sodium hypochorite solution soak ( $20 \mathrm{ml}$ household bleach to $980 \mathrm{ml}$ of water or 1 part bleach added to 49 parts water), which is an approved low-level disinfectant, for 10 minutes. In a previous version of this protocol, we had suggested $0.5 \%$ sodium hypochorite ( 1 part bleach to 9 parts water) which remains an approved high-level disinfectant.

Some suggest using only disinfectant wipes while others suggest a diluted bleach soak. Health Canada recommends cleaning with soap and water then disinfecting using an approved hard surface disinfectant with a drug identification number (DIN), or alternatively, a bleach soak. ${ }^{4}$ The theoretical advantage of the bleach soak is that it will penetrate the hinges and other small parts of the eye protection, and it conserves disinfectant wipes which may be in short supply. Health Canada states that eyewear may be disinfected (after appropriate cleaning with soap and water) using a $0.1 \%$ sodium hypochlorite solution soak (20 $\mathrm{ml}$ household bleach to $980 \mathrm{ml}$ of water or 1 part bleach added to 49 parts water), which is an approved low-level disinfectant, for 10 minutes. ${ }^{4}$ For efficacy, a new $0.1 \%$ sodium hypochlorite solution must be mixed every 24 hours.

\section{Limitations}

Our protocol has limitations as it assumes 3 important things; first, that the user is acquainted with and has applied jurisdictional policies and guidelines on when to use and how to manage PPE, second, it presupposes the health care clinician knows how to undertake good hand hygiene as per expert protocols and the sequences and safe donning and doffing of PPE, and third, it assumes the availability of PPE. Equipment shortages have occurred in many jurisdictions and strategies 
to conserve PPE have been required. Furthermore, these consensus recommendations represent expertise embodied in the workgroup and input from webinar participants. There are other insights and expertise that could apply and be customized for special situations.

\section{CONCLUSIONS}

This protocol provides a resource for health care professionals providing home visits when PPE is needed as per the jurisdictional policies on when to use PPE. It addressed a major gap in COVID-19 related guidelines, specifically the full process of donning and doffing PPE when doing home visits. It supplements jurisdictional guidelines and protocols on the criteria for when and how to use PPE. This resource, in the interim, serves as a useful procedure for health care professionals providing home-based care when PPE is required. Readers are invited to provide further input to assist in the ongoing work of improving and modifying the protocol to align with new knowledge emerging about the virus. Research is also needed to further study the safety of this and other similar protocols, and their various components.
To read or post commentaries in response to this article, go to https://www. AnnFamMed.org/content/19/5/405/tab-e-letters.

Key words: personal protective equipment; home care

Submitted August 11, 2020; submitted, revised, November 23, 2020; accepted December 3, 2020.

Acknowledgments: We wish to thank Rebecca Clark in the Research Office of the Department of Family Medicine at McMaster University for her assistance in editing and submitting the manuscript.

\section{References}

1. Palliative Care Division McMaster University. Resources. Updated 2020. https://fhs.mcmaster.ca/palliativecare/COVID19Resources.html

2. Global News. Coronavirus outbreak: WHO official says countries must rely on speed, not perfection in response. Published Mar 13, 2020. Accessed Jun 30, 2020. https://globalnews.ca/ video/6673069/coronavirus-outbreak-who-official-says-countriesmust-rely-on-speed-not-perfection-in-response

3. Ontario Health. Personal protective equipment (PPE) use during COVID-19 pandemic. Published May 10, 2020. https://www.ontario health.ca/sites/ontariohealth/files/2020-05/Ontario\%20Health\%20 Personal\%20Protective\%20Equipment\%20Use\%20During\%20 the\%20COVID-19\%20Pandemic_rev10May20\%20PDF_V2.pdf

4. Government of Canada. Updated: Public Health Management of cases and contacts associated with coronavirus disease 2019 (COVID-19). Published Apr 10, 2020. Updated Dec 23, 2020. https://www.canada.ca/en/public-health/services/diseases/2019novel-coronavirus-infection/health-professionals/interim-guidancecases-contacts.html

\section{Get the Annals of \\ FAMVILY MEDICINE} Family Medicine by E-mail

Make sure you see every new issue

while it's fresh; have the table of

contents sent to you by e-mail for

easy access to articles of interest

Don't miss important research.

Request the e-mail table of contents at https://www.annfammed.org/ JANUARY/FEBRUARY 2021 • Vol 19, No. 1

Editorial | Original Research | Research Briefs I Special Reports । Reflections Innovations I Departments

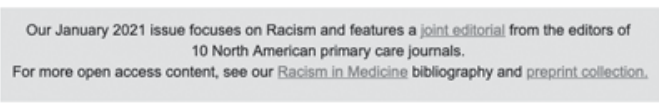

For more open access content, see our Bacion primary care joumats.

The full text of the journal is available online at wmw. AnnFammed.org. and through various aggregators, including PubMed Central, EBSCO, and ClinicalKey. The Annals is indexed in the MEDLINE, Science Citation Index Expanded, Current Contents/Clinical Medicine, EMBASE, and CINAHL databases.

\section{EDITORIAL}

Systemic Racism and Health Disparities: A Statement From Editors of Family Medicine Joumal: Sumi M. Sexton; Caroline R. Richardson; Sarina B. Schrager, Marjorie A. Bowman; John Hickner, Christopher P. Morley; Timothy F. Mott; Nicholas Pimlott; John W. Sauttz; Barry D. Weiss

The Annals and 10 other leading North American primary care journals pledge to confront the problem of racism in medicine by cultivating content that emphasizes cultural humility, diversity 8 inclusion, and the racism in molit bes reflections on the theme of systemic racism and health inequities.

\section{ORIGINAL RESEARCH}

ACC Discrimination and Medical Mistrust in a Racially and Ethnically Diverse Sample of Calffornia Adults Mohsen Bazargan; Sharon Cobb; Shervin Assar

The authors show that discrimination against patients, perceived or actual, is correlated with medical mistrust, and that decreasing discrimination may imorove trust in medical providers and decrease disparities in health 